EUROFUSION WPJET1-PR(16) 15001

C Angioni et al.

\title{
Gyrokinetic study of turbulent convection of heavy impurities in tokamak plasmas at comparable ion and electron heat fluxes
}

Preprint of Paper to be submitted for publication in Nuclear Fusion

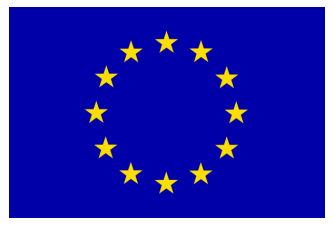

This work has been carried out within the framework of the EUROfusion Consortium and has received funding from the Euratom research and training programme 2014-2018 under grant agreement No 633053. The views and opinions expressed herein do not necessarily reflect those of the European Commission. 
This document is intended for publication in the open literature. It is made available on the clear understanding that it may not be further circulated and extracts or references may not be published prior to publication of the original when applicable, or without the consent of the Publications Officer, EUROfusion Programme Management Unit, Culham Science Centre, Abingdon, Oxon, OX14 3DB, UK or e-mail Publications.Officer@euro-fusion.org

Enquiries about Copyright and reproduction should be addressed to the Publications Officer, EUROfusion Programme Management Unit, Culham Science Centre, Abingdon, Oxon, OX14 3DB, UK or e-mail Publications.Officer@euro-fusion.org

The contents of this preprint and all other EUROfusion Preprints, Reports and Conference Papers are available to view online free at http://www.euro-fusionscipub.org. This site has full search facilities and e-mail alert options. In the JET specific papers the diagrams contained within the PDFs on this site are hyperlinked 


\title{
Gyrokinetic study of turbulent convection of heavy impurities in tokamak plasmas at comparable ion and electron heat fluxes
}

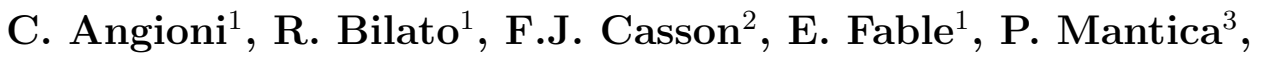 \\ T. Odstrcil ${ }^{1}$, M. Valisa ${ }^{4}$, ASDEX Upgrade Team and JET \\ contributors ${ }^{5} \ddagger$ \\ ${ }^{1}$ Max-Planck-Institut für Plasmaphysik, D-85748 Garching bei München, Germany \\ 2 CCFE, Culham Science Centre, Abingdon, OX14 3DB, UK \\ 3 Istituto di Fisica del Plasma, CNR/ENEA, Milano, Italy \\ ${ }^{4}$ Consorzio RFX-CNR/ENEA, I-35127 Padova, Italy \\ ${ }^{5}$ EUROfusion Consortium, JET, Culham Science Centre, Abingdon, OX14 3DB, UK
}

\begin{abstract}
In tokamaks, the role of turbulent transport of heavy impurities, relative to that of neoclassical transport, increases with increasing size of the plasma, as clarified by means of general scalings, which use the ITER standard scenario parameters as reference, and by actual results from a selection of discharges from ASDEX Upgrade and JET. This motivates the theoretical investigation of the properties of the turbulent convection of heavy impurities by nonlinear gyrokinetic simulations in the experimentally relevant conditions of comparable ion and electron heat fluxes. These conditions also correspond to an intermediate regime between dominant ion temperature gradient turbulence and trapped electron mode turbulence. At moderate plasma toroidal rotation, the turbulent convection of heavy impurities, computed with nonlinear gyrokinetic simulations, is found to be directed outward, in contrast to that obtained by quasi-linear calculations based on the most unstable linear mode, which is directed inward. In this mixed turbulence regime, with comparable electron and ion heat fluxes, the nonlinear results of the impurity transport can be explained by the coexistence of both ion temperature gradient and trapped electron modes in the turbulent state, both contributing to the turbulent convection and diffusion of the impurity. The impact of toroidal rotation on the turbulent convection is also clarified.
\end{abstract}

PACS numbers: 52.25.Fi, 52.55.Fa, 52.65.Tt

$\ddagger$ See the Appendix of F. Romanelli et al., Proceedings of the 25th IAEA Fusion Energy Conference 2014, Saint Petersburg, Russia 


\section{Introduction and motivation}

In tokamaks, heavy impurities are transported by both neoclassical and turbulent processes. Central accumulation is the consequence of the dominance of neoclassical transport under conditions where main ion density profiles are centrally peaked and ion temperature profiles are not peaked enough to compensate by temperature screening. Recent and past analyses of accumulation of highly charged impurities have regularly identified the signature of neoclassical transport $[1,2,3,4,5,6,7,8,9,10,11,12,13]$. The deleterious impact of neoclassical transport can be removed or at least mitigated by an increase of turbulent transport. The latter can increase the turbulent diffusion, reducing the impact of the neoclassical convection $[8,14]$, but can also contribute by means of additional turbulent convection mechanisms, which are theoretically predicted $[15,16,17,18,19,20,21,22,23,24,25,26]$. Over the last decade, several works have been also dedicated to the comparison between the theoretical predictions of turbulent impurity transport and the experimental measurements $[27,28,29,30,31,32,33,34$, 35, 36]. Conditions where turbulent diffusion dominates over the neoclassical diffusion can be achieved in present devices, particularly by means of the application of central heating. Electron heat fluxes slightly exceeding the ion heat fluxes are predicted to maximize the turbulent diffusion for a given total heat flux [37]. In contrast, conditions where the turbulent convection of heavy impurities dominates over the neoclassical convection are difficult to achieve in present devices, due to both the proportionality to the impurity charge of the neoclassical convection [40] and the enhancement of neoclassical transport produced by poloidal asymmetry of the impurity density, as induced by plasma rotation $[41,42,43,44,45]$. Nevertheless, the conditions which can be expected to be achieved in future fusion reactors are different. Not only rotation should be expected to be significantly lower than in present experiments with large external torque by neutral beam injection (NBI), but also the plasma will achieve a collisional regime in which neoclassical transport is relatively lower than in present experiments. Moreover, the stronger magnetic field reduces the poloidal Larmor radius. This is exemplified in Fig. 1, where the ratio of the neoclassical heat flux to the total heat flux around $\rho \simeq 0.3$ is plotted as a function of the heating power for a set of plasmas obtained in devices of increasing size with parameters determined by appropriately rescaling those of the ITER standard scenario, that is keeping constant the ratios $R / a$,

$I_{p} / a^{2}, R B_{T} / I_{p}, n / n_{G W}$ and assuming a confinement time as given by the IPB98 scaling law [46] (here $R$ and $a$ are major and minor radius, $I_{p}$ is the plasma current, $B_{T}$ is the toroidal magnetic field and $n_{G W}$ is the Greenwald density limit). It is also assumed that half of the heating power is deposited inside $\rho \simeq 0.3$, which is approximately fulfilled by central NBI and by $\alpha$ heating power density profiles. By computing the fraction of the heat flux which is carried by neoclassical transport, in Fig. 1(a) we observe that, due to the increase of plasma confinement with increasing size and current, and therefore due to the higher temperatures in a reactor, a reactor size plasma achieves conditions of significantly lower ratios of neoclassical transport to total transport at constant ratio of the density to the density limit. We identify the operational points 
Gyrokinetic study of turbulent convection of heavy impurities in tokamak plasmas at comparable ion and
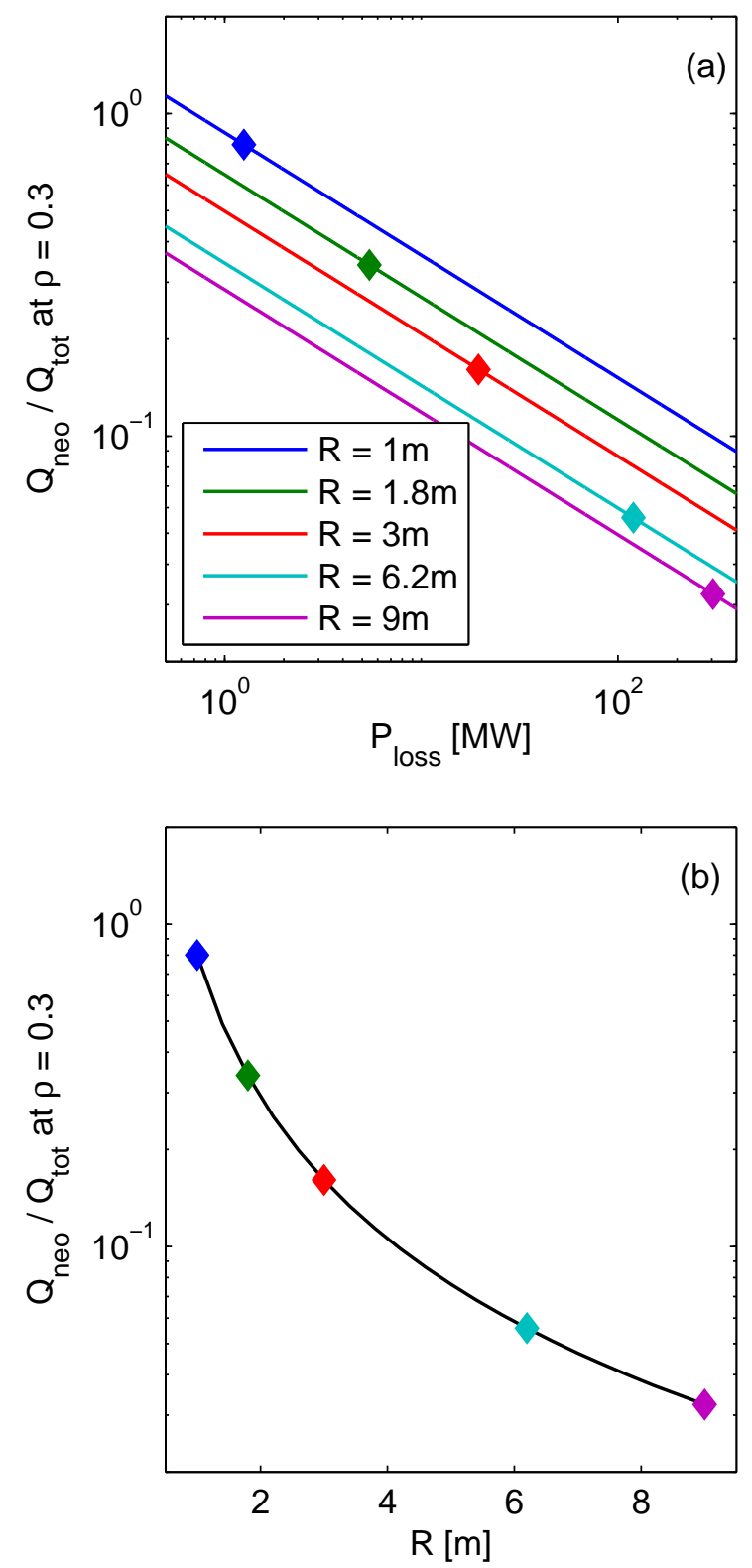

Figure 1. Neoclassical to total heat flux ratio estimated at $\rho_{\phi}=0.3$ as a function of the total loss power for plasmas of different size (a), and as a function of the major radius $R$ at the total loss powers presented with full symbols in (a), corresponding to $P_{\text {loss }} \propto R^{2.5}$. ITER parameters ( $R=6.2 m$ in the legend) are scaled following the prescriptions of constant $R / a, I_{p} / a^{2}, R B_{T} / I_{p}, n / n_{G W}$. 


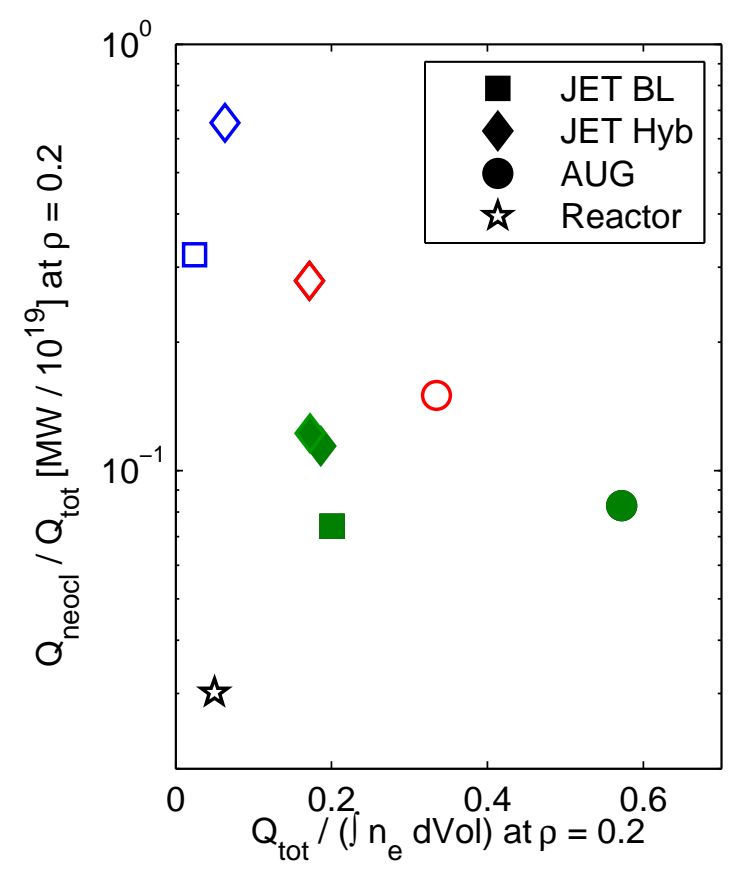

Figure 2. Neoclassical to total heat flux ratio estimated at $\rho_{\phi}=0.2$ as a function of the total heating power per particle inside the same radius $\rho_{\phi}=0.2$. JET H-mode plasmas in baseline scenario are plotted with squares, JET H-mode plasmas in hybrid scenario are plotted with diamonds, AUG H-modes are plotted with circles. Plasmas without central RF heating are plotted with open symbols (with NBI heaeting only in blue, with NBI and predominantly off-axis RF heating in red). Plasmas with central $\mathrm{RF}$ heating are plotted with full symbols (dark green). Reference conditions of a reactor (DEMO) are plotted with a star.

of the plasmas with increasing size by considering a total loss power $P_{\text {loss }}$ proportional to $R^{2.5}$, which reasonably reproduces the increase of the heating power usually applied from small to large devices (this does not correspond to constant $\beta$ conditions). In Fig. $1(\mathrm{~b})$, the decrease of the estimated ratio $Q_{\text {neo }} / Q_{r m t o t}$ is plotted as a function of the increasing plasma size. The curve shows that neoclassical transport has a larger weight in present devices than in future reactor size plasmas. This is clearly a favorable scaling with respect to the threat of heavy impurity accumulation. This also implies that with increasing size of the device the requirements in terms of central localization of the heating power density profile in order to increase the turbulent transport component are reduced, that is in a bigger device the same ratio of the neoclassical flux to total heat flux can be obtained with lower levels of heating power per partcle in the central region of the plasma.

These general results obtained from the application of global scalings are confirmed by the analysis of a selection of JET and ASDEX Upgrade (AUG) discharges on which detailed power balance analyses have been performed, combined with specific neoclassical transport calculations. The results, presented in Fig. 2, show that in the core the neoclassical transport component is reduced by increasing the heating power per particle, and that similar values of neoclassical transport fraction are obtained in a 
larger device (JET) at significantly smaller values of power per particle than in a smaller device (AUG). Indeed AUG has to reach a heating power of $0.6 \mathrm{MW}$ per $10^{19}$ particles in order to achieve the same central neoclassical heat flux fraction of JET with only one third of the power per particle. Interstingly, among these selected experimental cases, all of those which do not have sufficient additional central radiofrequency (RF) heating (ion cyclotron at JET and electron cyclotron at AUG) exhibit $\mathrm{W}$ accumulation during the development of the discharge (open symbols in Fig. 2). A detailed description of the JET discharges can be found in [11] (hybrid scenario H-mode with NBI only $\sharp 82722$ ), in [13] (baseline scenario H-mode with NBI only, $\sharp 83351$ ), and $[47,12,48]$ (hybrid scenario H-modes with NBI and ICRH on-axis $\sharp 82753$ and $\sharp 86871$ and off-axis $\sharp 82752$, as well as baseline scenario H-mode with NBI and ICRH on-axis $\sharp 85307)$. The two AUG points are taken from usual AUG $\mathrm{H}$-modes at $2.5 \mathrm{~T}$ and $1 \mathrm{MA}$, with $7.5 \mathrm{MW}$ of NBI power and 2.1 MW of ECH power on-axis (full circle), 0.7 MW on-axis and 1.4 MW off-axis (open circle). Moreover, consistent with the general scaling presented in Fig. 1, in the plasma of a proposed reactor (DEMO) $[49,50]$ a much lower fraction of neoclassical heat flux is obtained, despite of the low levels of heating power per partilce. In conclusion, the scaling presented in Fig. 1 and the results of Fig. 2 clearly motivate a careful study of the turbulent transport of heavy impurities for the prediction of the impurity density profiles in proposed scenarios of reactors, also taking into consideration the need of controlling and optimizing the profiles of the radiated power density produced by impurity seeding, with the aim to reduce divertor heat loads without reducing the plasma stored energy.

These arguments have motivated the present work dedicated to the simulation of the convection of tungsten (W) by means of nonlinear gyrokinetic simulations in which the turbulent state of the plasma produces comparable electron and ion heat fluxes. Comparable electron and ion heat fluxes can be expected to be present in the plasma of a fusion reactor, where the ratio of the thermal collisional exchange time to the confinement time is small. These are also the conditions where, at constant total turbulent heat flux, the turbulent diffusion of heavy impurities is theoretically predicted to be the largest [37]. In this work we extend that recent study with the gyrokinetic code GKW $[38,39]$ and we investigate the nonlinear properties of the turbulent convection of W. These are presented in Section 2. By direct comparison with quasi-linear predictions based on the most unstable linear mode, we find that $\mathrm{W}$ convection is directed outward in the nonlinear result and inward for the most unstable linear mode. Previous nonlinear gyrokinetic studies dedicated to intermediate regimes between ion temperature gradient (ITG) and trapped electron mode (TEM) turbulence [51] motivate the inspection of the nature and the transport of subdominant linear modes as possible explanation of the observed differences in the $\mathrm{W}$ convection. Moreover, a possible non-negligible role of subdominant modes on turbulent impurity transport was previously considered in an attempt of reducing the disagreement between the gyrokinetic predictions based on the dominant linear mode and the observed nickel convection in impurity transport experiments at JET [27]. In Section 3 we show that the difference between the nonlinear results and the linear results provided by the dominant linear mode can be explained 
by the coexistence of both ion temperature gradient and trapped electron modes in the nonlinear turbulent state, both contributing to the turbulent convection of the impurity. Thereby, in these conditions the impurity convection plays the role of a macroscopic fingerprint of the co-existence of both ITG and TEM in the turbulent state described by the nonlinear gyrokinetic simulation. Moreover, the turbulence regimes (and the corresponding electron to ion heating ratios) over which turbulent convection of heavy impurities is predicted to be directed outwards are more extended in nonlinear gyrokinetic simulations than in single mode quasi-linear models. Thereby they require an adequate combination of the contributions of dominant and sub-dominant modes for a correct prediction by means of a quasi-linear model. In section 4 , the impact of toroidal rotation is examined, and it is shown to lead to an increase of outward convection, which is an unfavorable element in the projection to a fusion reactor, with expected lower levels of toroidal rotation than in present devices, which are usually operated with substantial external torque.

\section{Nonlinear gyrokinetic simulations of $W$ convection at moderate toroidal rotation}

The nonlinear gyrokinetic simulations have been performed with the gyrokinetic code GKW [38, 39] and have extended those presented in [37] to the calculation of all of the convective contributions. These can be decomposed in the following form $[16,20,25]$

$$
\frac{R \Gamma_{W}}{n_{W}}=D_{N} \frac{R}{L_{n W}}+D_{T} \frac{R}{L_{T}}+D_{U} u^{\prime}+R V_{p}
$$

where $R / L_{n}=-(R / n)(d n / d r), R / L_{T}=-(R / T)(d T / d r)$ and $u^{\prime}=-\left(R^{2} / v_{t h W}\right) d \Omega / d r$, where $\Omega$ is the $\mathrm{W}$ toroidal rotation, and where we refer to the low field side density in the presence of toroidal rotation and consequent poloidal inhomogeneity of the $\mathrm{W}$ density.

We simulate local plasma conditions around the normalized minor radius $r / a=$ 0.35 , and we consider local inverse aspect ratio $\epsilon=0.105$, local safety factor $q=1.29$ and magnetic shear $\hat{s}=0.31$, and reference experimental values of the local electron density $n_{e}=7.010^{19} \mathrm{~m}^{-3}$ and local ion temperature $T_{i}=3.8 \mathrm{keV}$, local thermal deuterium (D) Mach number of the toroidal rotation $M_{D}=0.166$ and normalized logarithmic density gradient $R / L_{n}=2.0$, as observed in a reference AUG plasma, from which also the magnetic equilibrium geometry as provided by a numerical equilibrium reconstruction, is taken as input. Similarly to the work presented in Ref. [37]. the electron and ion temperature logarithmic gradients and the electron to ion temperature ratio have been varied in order to vary the ion to electron heat flux keeping fixed the total heat power. This allows us to explore conditions where the ratio $Q_{e} / Q_{i}$ varies by two orders of magnitude (from 0.15 to 16 ) and where the corresponding turbulence is produced by ITG modes and progressively transitions to turbulence produced by TEM. The parameters used in the simulation are reported in Table 1, where the results related to $\mathrm{W}$ convection have been included, (the reader is referred to Table 1 of Ref. [37] for the results related to the $\mathrm{W}$ diffusion). All the nonlinear simulations have been performed with 43 toroidal 
Gyrokinetic study of turbulent convection of heavy impurities in tokamak plasmas at comparable ion and

\begin{tabular}{cccccccc} 
& $R / L_{T i}$ & $R / L_{T e}$ & $T_{e} / T_{i}$ & $Q_{e}+Q_{i}$ & $Q_{e} / Q_{i}$ & $R V_{p W} / \chi_{i}$ & $R V_{p W} / D_{W}$ \\
\hline $\mathrm{A}$ & 1.0 & 13.0 & 1.900 & 12.97 & 15.98 & 0.907 & 3.367 \\
$\mathrm{~B}$ & 3.0 & 12.0 & 2.000 & 13.06 & 7.500 & 2.052 & 2.648 \\
$\mathrm{C}$ & 4.0 & 12.0 & 1.600 & 13.23 & 1.731 & 2.296 & 1.087 \\
$\mathrm{D}$ & 4.0 & 12.0 & 1.500 & 11.75 & 1.590 & 2.068 & 1.003 \\
$\mathrm{E}$ & 5.0 & 12.0 & 1.175 & 13.22 & 1.034 & 0.734 & 0.551 \\
$\mathrm{~F}$ & 6.0 & 6.0 & 1.000 & 13.77 & 0.345 & 0.029 & 0.077 \\
$\mathrm{G}$ & 7.0 & 4.0 & 0.900 & 13.50 & 0.221 & -0.005 & -0.018 \\
$\mathrm{H}$ & 8.0 & 2.0 & 0.850 & 12.90 & 0.153 & 0.003 & 0.015
\end{tabular}

Table 1. Local parameters which have been varied as inputs of the nonlinear gyrokinetic simulations and corresponding results obtained. Total heat flux is expressed in gyroBohm units and normalized to $n_{e} T_{i} v_{t h i}\left(\rho_{i} / R\right)^{2}$. At the local AUG plasma parameters considered as experimental reference, a value of the total heat flux of 13 in normalized units corresponds to a flux of 2.175 MW.

modes and 339 radial modes, with a box size in the binormal and radial directions given by $L_{y}=94.25 \rho_{i}$ and $L_{x}=80.45 \rho_{i}$, with $\rho_{i}=\left(2 m T_{i}\right)^{0.5} / B$ (minimum non-zero and maximum values of corresponding wave numbers are $k_{y} \rho_{i}=0.0667, \operatorname{abs}\left(k_{x} \rho_{i}\right)=0.0702$ and $k_{y} \rho_{i}=2.800, \operatorname{abs}\left(k_{x} \rho_{i}\right)=11.8663$ respectively). We used a grid of 16 points in one turn along the field line, and the velocity space is discretized over 32 points in the parallel velocity and 8 points in the magnetic moment. The collision operator applied in these simulations includes pitch-angle scattering only.

We remind that when the impurity is in the trace limit, the flux is linear in the impurity gradients, also in nonlinear simulations. This allows a non-ambiguous decomposition of the different off-diagonal contributions [52]. This decomposition has been performed in a set of nonlinear simulations where trace $\mathrm{W}$ species have been included with a charge $Z_{W}=41\left(A_{W}=184\right)$. A companion set of linear simulations has been also carried out at a single binormal wave number $k_{y} \rho_{i}=0.4$ where the nonlinear spectrum of the $\mathrm{W}$ transport is found to peak, in order to directly compare nonlinear results with the linear results. Fig. 3 summarizes the results obtained for the pure convection $V_{p W}$ of $\mathrm{W}$ (last term on the right hand side of Eq. (1), that is, the off-diagonal component which is not proportional to any gradient of any $\mathrm{W}$ kinetic profile). The pure convection $R V_{p W}$ (multiplied by the major radius $\mathrm{R}$ ) is shown normalized to the ion and electron heat conductivities $\chi_{i}$ and $\chi_{e}$, to the effective heat conductivity $\chi_{\mathrm{eff}}=-\left(q_{i}+q_{e}\right) /\left(n_{e} d T_{e} / d r+n_{i} d T_{i} / d r\right)$, as well as to the $\mathrm{W}$ diffusion coefficient $D_{W}$. We observe that the pure convection is directed outward when $Q_{e} \gg Q_{i}$, in dominant TEM turbulence, whereas it is very small (and directed inward) when $Q_{e} \ll Q_{i}$, in dominant ITG turbulence. This is at least qualitatively consistent with the linear results, and largely consistent with expected dependencies in the different turbulence regimes from previous studies [26]. However we also observe that for the experimentally relevant conditions of $Q_{e} \simeq Q_{i}$ linear and nonlinear results are in opposite directions. One might suspect that these differences come from spectral components in the nonlinear simulations which are not accounted for in a single wave number linear 

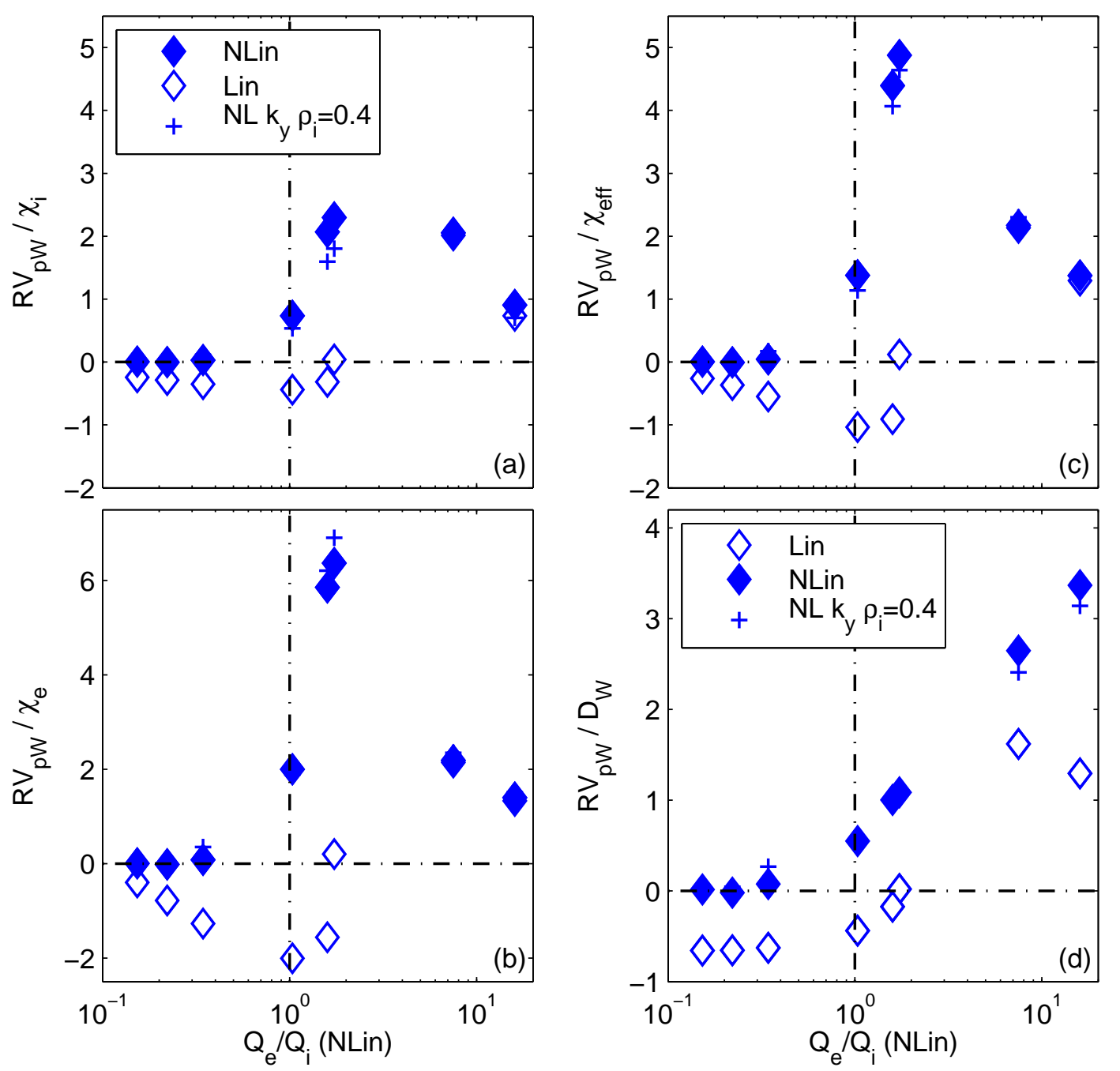

Figure 3. Pure convection of $\mathrm{W}$ normalized to normalized to the ion, electron and effective heat conductivities $\left(\chi_{i}(\mathrm{a}), \chi_{e}(\mathrm{~b})\right.$ and $\chi$ eff $\left.(\mathrm{c})\right)$ as well as to the $\mathrm{W}$ diffusion coefficient $D_{W}$, plotted as a function of the electron to ion heat flux ratio as obtained in the nonlinear simulations. Nonlinear results are plotted with full diamonds, linear results at $k_{y} \rho_{i}=0.4$ obtained from the most unstable linear mode are plotted with open diamonds. Finally, crosses + show the nonlinear results at $k_{y} \rho_{i}=0.4$, that is the same mode number of the linear runs, where the nonlinear spectrum peaks.

calculation. However, this should be also reflected in the fact that nonlinear results considered at the same single wave number $k_{y} \rho_{i}=0.4$ should significantly differ from the complete nonlinear results obtained by summation over a wide spectrum of wave numbers. The nonlinear results at $k_{y} \rho_{i}=0.4$ are shown with crosses + and are in almost perfect agreement with the complete nonlinear results, which demonstrates that the contribution at the peak of the nonlinear spectrum is indeed dominant and that differences between linear and nonlinear results cannot be explained by contributions at different $k_{y}$ numbers. 
Gyrokinetic study of turbulent convection of heavy impurities in tokamak plasmas at comparable ion and
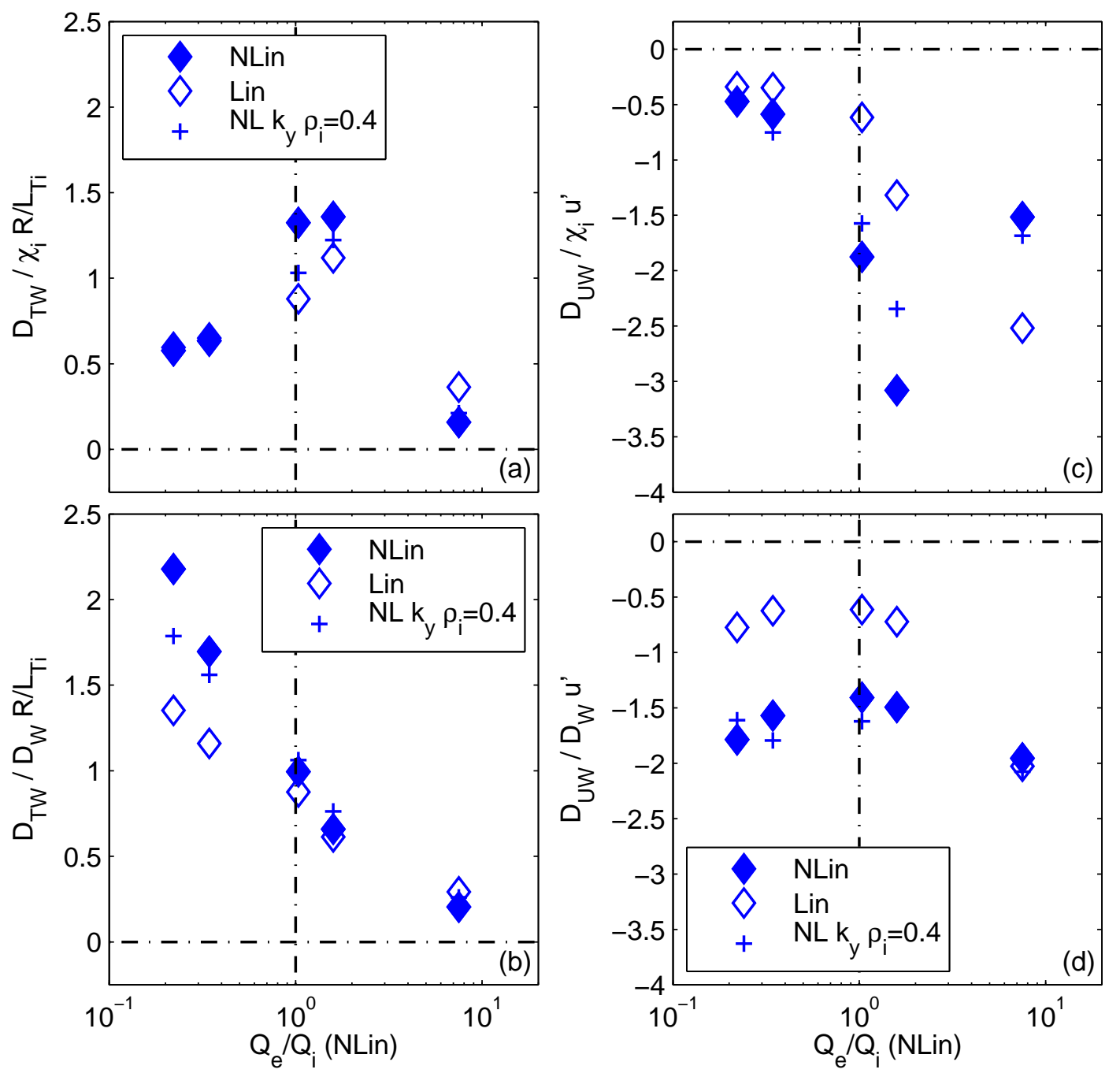

Figure 4. Thermo-diffusion contribution $D_{T} R / L_{T i}(\mathrm{a}, \mathrm{b})$ and roto-diffusion contribution $(\mathrm{c}, \mathrm{d})$ normalized to the ion heat conductivity $\chi_{i}(\mathrm{a}, \mathrm{c})$ and to the $\mathrm{W}$ diffusion coefficient $D_{W}(\mathrm{~b}, \mathrm{~d})$ respectively, plotted as a function of the electron to ion heat flux ratio as obtained in th nonlinear simulations. Symbols are the same as in Fig. 2.

Fig. 4 shows the additional off-diagonal contributions related to the ion temperature gradient (Fig. $4 \mathrm{a}, \mathrm{b}$ ) and to the radial gradient of the toroidal angular velocity (Fig. 4 c,d). Both transport coefficients do not show strong differences between nonlinear results and linear results, as obtained from the most unstable mode at a single wavenumber, in contrast to the results presented for the pure convection in Fig. 3 (in fact, as it will be clear from the results presented in the next section, the main differences from nonlinear and linear results are due to differences in the normalization coefficients, ion heat conductivity $\chi_{i}$ and diffusion coefficient $D_{W}$ ).

In the next section, we examine in detail case D of Table 1 , with $Q_{e} / Q_{i}=1.590$, which exhibits the largest differences between nonlinear and linear results of the $\mathrm{W}$ convection $R V_{p W}$. 


\section{Role of dominant and subdominant modes on the turbulent convection of heavy impurities}

The eigenvalue solver of GKW [53] has been applied to identify possible subdominant modes at the conditions of case $\mathrm{D}$ in table 1 , with $Q_{e} / Q_{i}=1.590$. The eigenvalues (real and imaginary parts) are presented in Fig. 5 as a function of the binormal wave number, together with the corresponding spectra of the growth rate (imaginary part) and real frequency (real part) of the most unstable linear mode, as calculated by the initial value (time integration) linear solver of the GKW code, which identifies only the most unstable linear mode. We observe that, under these conditions, in the interesting spectral domain around $k_{y} \rho_{i}=0.4$ where the maximum transport takes place, as shown by the nonlinear spectrum in Fig. 5(c), two modes are unstable, with close growth rates, plotted in Fig. 5(a), but with opposite real frequencies, plotted in Fig. 5(b), corresponding to an ITG mode, which is dominant for $k_{y} \rho_{i}<0.75$ and a TEM mode, which is subdominant for $k_{y} \rho_{i}<0.75$ and becomes dominant for $k_{y} \rho_{i}>0.75$.

The spectra of the contributions to the transport of W, as described by Eq. (1), are presented in Fig. 6, normalized to the amplitude squared of the electrostatic potential fluctuations $\left|\phi_{k}\right|^{2}$, providing a direct comparison between nonlinear and linear results. The pure convection term, which has motivated this investigation, is plotted in Fig. 6(a). Consistent with the results presented in Section 2, we observe that at $k_{y} \rho_{i} \simeq 0.4$ the nonlinear results (full diamonds) are positive, whereas the linear results, obtained from a single dominant mode (open triangles pointing up) are small (in absolute value) and negative. These linear results correspond to those of the dominant ITG mode, as also identified by the eigenvalue solver (full triangles pointing left). The subdominant (at $\left.k_{y} \rho_{i} \simeq 0.4\right) \mathrm{TEM}$ (full triangles pointing right) produce a stronger convection directed outward. We observe that, around $k_{y} \rho_{i}=0.4$, the nonlinear spectrum is practically in the middle between the convection produced by the small negative (dominant) ITG mode, and the large positive (subdominant) TEM. This suggests that the nonlinear transport can be described by a combination of contributions from dominant and subdominant linear modes, performing an average which is weighted by means of an appropriate power of the corresponding growth rates. In a previous work, where intermediate turbulence regimes were investigated, the role of subdominant modes was investigated for heat transport [51]. Subdominant modes are also considered in quasilinear transport models, in which multiple eigenvalues are computed from the fluid $[54,55]$ or kinetic [56] dispersion relation. Here, we apply these concepts to impurity transport and we check whether a quasi-linear model based on multiple eigenvalues can explain and even quantitatively reproduce the nonlinear results. To this end, we propose the following generic expression for the transport coefficients, which introduces a weighted average among dominant and subdominant modes,

$$
W_{\mathrm{ave}, \mathrm{k}}=\frac{W_{A k} \gamma_{A k}^{p}+W_{B k} \gamma_{B k}^{p}}{\gamma_{A k}^{p}+\gamma_{B k}^{p}}
$$

In Eq. (2), $W_{A k}, W_{B k}$ are generic quasi-linear transport weights, that is quasi-linear radial fluxes $\left\langle\tilde{n}_{k} \tilde{v}_{k E \times B}\right\rangle$ computed at a single wave number $k_{y}$ and divided by the 
Gyrokinetic study of turbulent convection of heavy impurities in tokamak plasmas at comparable ion and

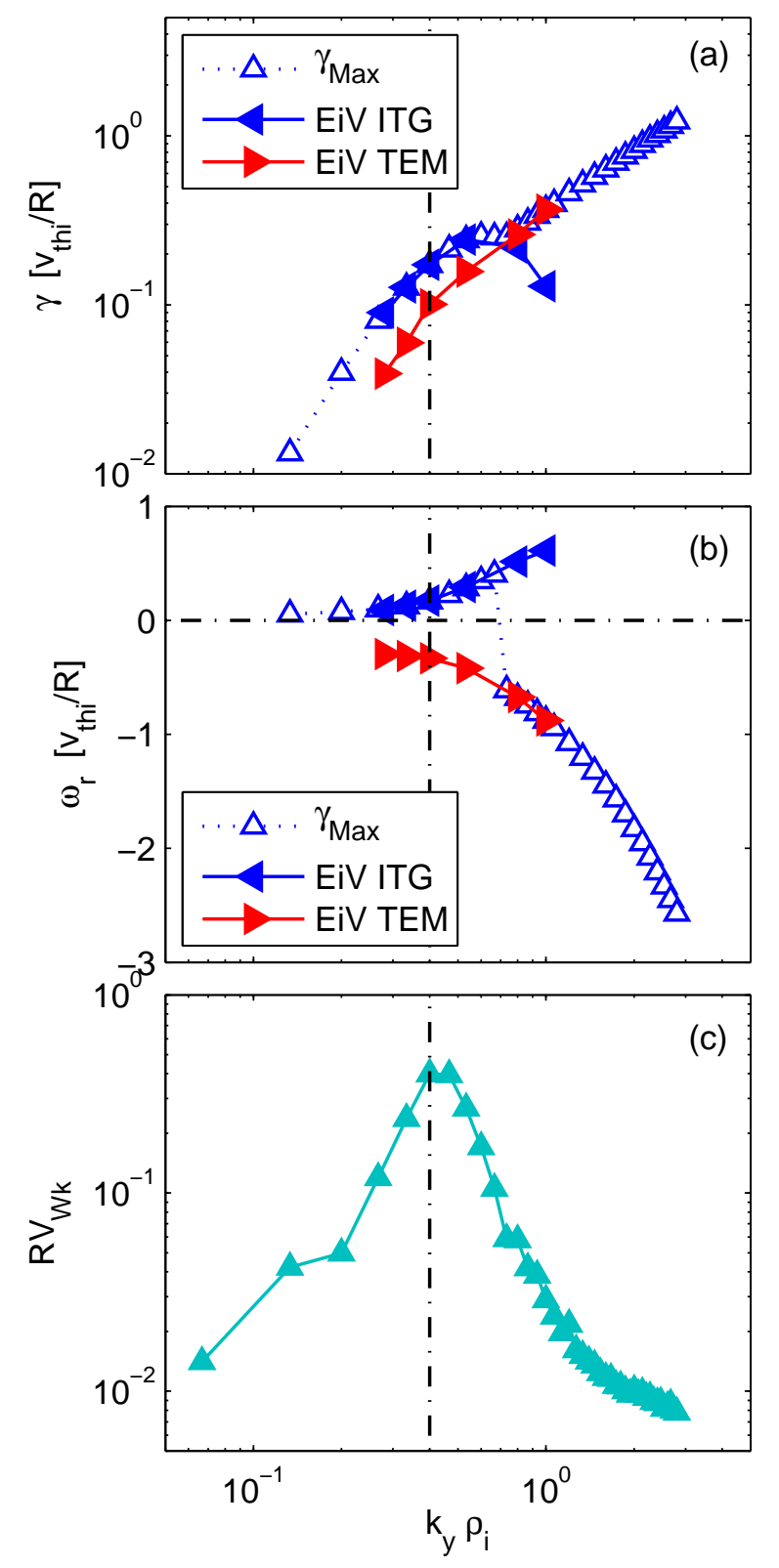

Figure 5. Spectra of the linear growth rate (a), linear real frequency (b) and nonlinear spectrum of the $\mathrm{W}$ pure convection in the nonlinear simulation (c) as a function of the binormal wave number $k_{y} \rho_{i}$. In (a,b), fastest growing linear modes are plotted with open triangles pointing up, ITG mode and TEM obtained by the eigenvalue solver (EiV in the legend) are plotted with full triangles pointing left and right respectively. 

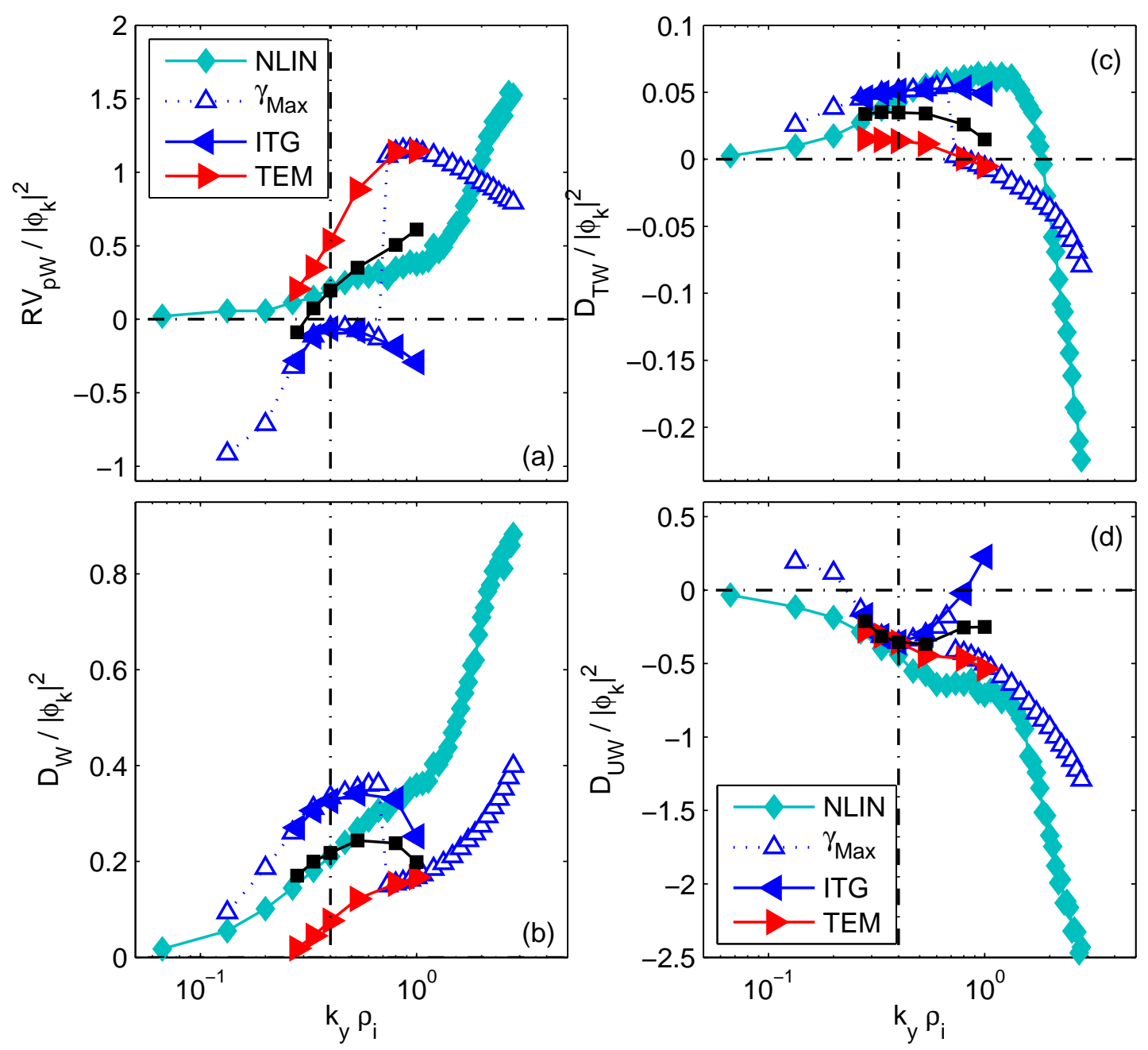

Figure 6. Spectra of the transport weights of the pure convection (a), of the diffusion (b), of the thermo-diffusion (c) and of the roto-diffusion (d) as a function of the binormal wave number $k_{y} \rho_{i}$. Full diamonds are the nonlinear results, open triangles pointing up are the linear results obtained from the fastest growing linear mode, full triangles pointing left are the linear results obtained from the ITG mode and full triangles pointing right are the linear results obtained from the TEM, computed by the eigenvalue solver (EiV in the legend). Finally, full squares are the linear results obtained by the mode average described by Eq. (2).

corresponding electrostatic potential squared $\left|\phi_{k}\right|^{2} . \quad W_{A k}$ and $W_{B k}$ correspond to dominant and subdominant linear modes, with growth rates $\gamma_{A k}$ and $\gamma_{B k}$ respectively. The resulting averaged quasi-linear transport weight $W_{\text {ave, } \mathrm{k}}$ is obtained by averaging the contributions of the two modes, with a weighting factor given by the growth rates to power $p$. We have varied the exponent $p$ in order to identify the value which most closely matches the nonlinear results in the spectral range where the nonlinear transport peaks, that is around $k_{y} \rho_{i}=0.4$. We have found that the value $p=0.5$ provides the best match, but good fits of the nonlinear results are also obtained with $p=1$, which is usually adopted in quasi-linear models (clearly worse is the choice $p=2$ ). 
The proposed quasi-linear model combining contributions from dominant and subdominant modes of Eq. (2) is applied to the calculation of the other components of $\mathrm{W}$ transport in Fig. 6(b-d). The averaged quasi-linear transport weight is found to quantitatively reproduce the nonlinear results in the relevant $k_{y} \rho_{i}$ range, in particular correcting the differences between single dominant mode results and the nonlinear results found for the diffusion coefficient $D_{W}$, Fig. 6(b). Smaller differences between dominant and subdominant modes are found for the thermo-diffusion $D_{T W}$ and the roto-diffusion $D_{U W}$ transport coefficients, as shown in Fig. 6(c) and 6(d) respectively.

\section{Dependence on toroidal rotation}

It is of interest to investigate the dependence of the impurity transport as a function of the toroidal plasma rotation. In the previous sections we have considered a typical value of thermal Mach number of $\mathrm{D}$, that is $M_{D} \simeq 0.15$, measured in a moderately high density plasma with NBI heating in AUG. Predictions of toroidal rotation velocities in future reactors have large uncertainties, but can be expected not to exceed those observed in present devices with large NBI torques. This justifies to study the dependence of the impurity transport as a function of the toroidal plasma velocity. Moderate values of plasma velocity as achieved in conventional aspect ratio tokamaks have small impact on the bullk plasma, but, due to the much lower thermal velocity of $\mathrm{W}$, can have significant impact on the $\mathrm{W}$ transport [25]. In particular, they produce an outward contribution in the pure convective term (Eq. 26 and Fig. 1 of Ref. [25]). The effect of this contribution is clearly visible in the results presented in Fig. 7. At small values of the toroidal rotation (expected in a fusion reactor due to the small ratio of plasma inertia to injected torque), the reduction of the centrifugal outward component implies a pure convection which is directed inward and provided by the combination of an inward contribution from the dominant ITG mode (triangles pointing left) and a very small outward contribution provided by the subdominant TEM (triangles pointing right), still consistent with Eq. (2) (squares).

With increasing toroidal rotation, a regime of moderate rotation velocities is reached where dominant and subdominant modes have opposite directions, providing nonlinear results which are in opposite direction with respect to those of the linear mode, and which are correctly tracked by the quasi-linear rule Eq. (2). Finally, at sufficiently large toroidal velocities, both dominant ITG mode and subdominant TEM mode produce and outward convection of the heavy impurity, resulting in an outward convection as obtained both in the combined quasi-linear weights and in the nonlinear results. Analogously good comparisons between quasi-linear weights computed from Eq. (2) and the nonlinear weights obtained from actual nonlinear simulations are found for the diffusion coefficient.

While the favorable effect of outward convection is lost at low rotation velocities, the nonlinear results still provide a predicted convection which has slightly reduced values of $R V_{p W} / D_{W}$ at zero rotation with respect to the results provided by the dominant mode only. This still highlights the favorable effect of the subdominant mode. This also 
Gyrokinetic study of turbulent convection of heavy impurities in tokamak plasmas at comparable ion and
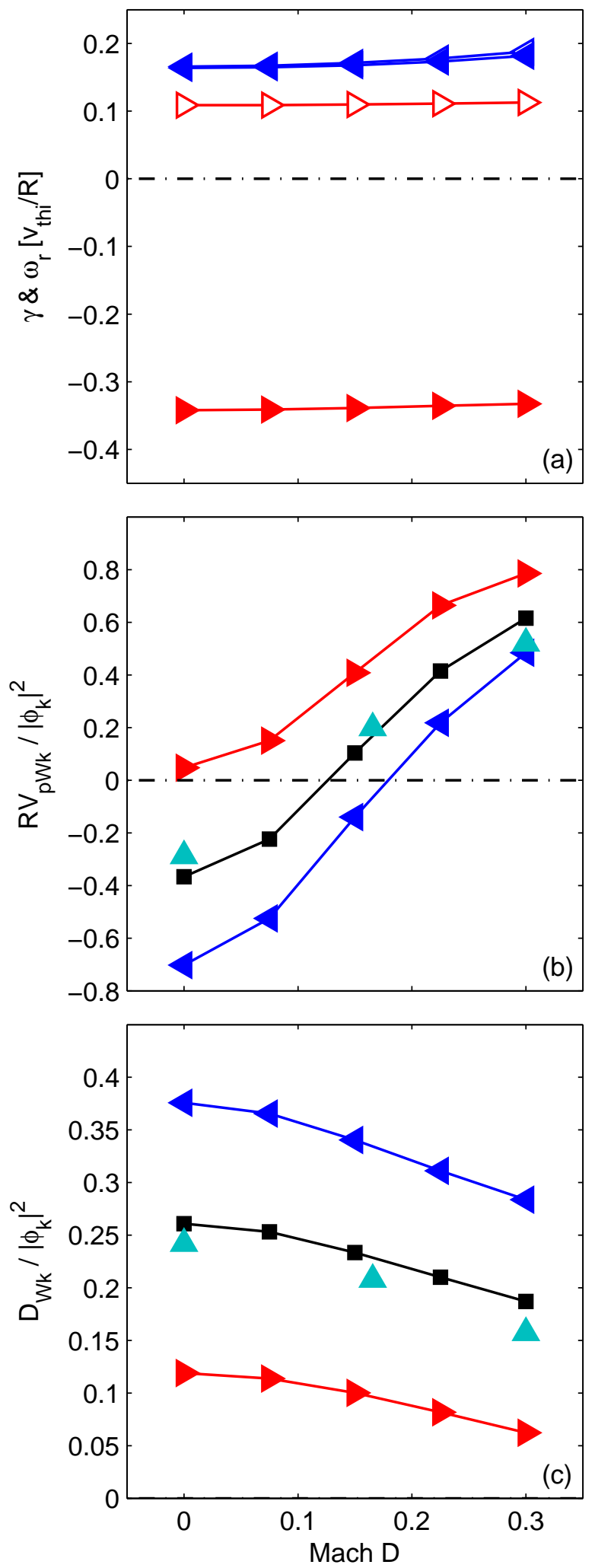

Figure 7. Linear growth rate (open symbols) and real frequency (full symbols0) (a) and transport weights of the pure convection (b) and the diffusivity (c) at $k_{y} \rho_{i}=0.4$ for the dominant ITG mode (triangles pointing left) and the subdominant TEM (triangles pointing right). The mode averages described by Eq. (2) (full squares) are compared with nonlinear results (full triangles pointing up) at selected Mach numbers (b,c). 
implies that, thanks to the contribution of the subdominant mode, the value of $M_{D}$ at which the $\mathrm{W}$ convection reverses sign from inward to outward is smaller in the nonlinear simulations than in the results obtained with the dominant linear mode alone.

\section{Conclusions}

In present tokamaks heavy impurity transport is more regularly dominated by neoclassical convection than in future tokamak reactors. Larger size and larger current imply better confinement, and therefore the achievement of higher temperatures at the same densities, which, also combined with the larger magnetic field, imply a reduced neoclassical transport. This trend is confirmed by actual neoclassical transport calculations combined with power balance analyses of a selection of AUG and JET plasmas, also compared to the results obtained for a reference reactor (DEMO) plasma. The expected increasing importance of the turbulent transport of heavy impurities in a reactor plasma motivates studies which characterize the properties of turbulent impurity convection, also with the aim of identifying the most appropriate parameter ranges for an effective validation of the theoretical predictions by means of dedicated experiments. The work presented in this paper builds on a previous study, which investigated the dependence of the turbulent diffusion coefficient of a heavy impurity like $\mathrm{W}$ as a function of the ratio of the electron to ion heat flux by means of linear and nonlinear gyrokinetic simulations [37]. Here, this gyrokinetic study has been extended, still with the GKW code, in order to investigate the properties of the turbulent convection of heavy impurities, with particular emphasis on the experimentally relevant conditions where the ion and electron heat fluxes are comparable. It is found that, in these conditions and at typical moderate toroidal rotation velocities, as measured in present devices with NBI heating at intermediate to high densities, the nonlinear results are qualitatively different from the linear ones based on the most unstable linear mode. The nonlinear simulations produce a relatively large outward $\mathrm{W}$ convection, whereas the linear results produce a small inward convection. Differences between nonlinear and linear results have been explored by investigating the role of subdominant linear modes in the nonlinear turbulent regime, by the application of the eigenvalue solver of the GKW code. Turbulence regimes which produce comparable ion and electron heat fluxes are regularly characterized by the co-existence of TEM and ITG modes in the spectral range of binormal wave numbers where the transport peaks [51]. A turbulence regime of this type has been specifically considered in this work in the framework of impurity transport. It is found that the outward convection obtained in the nonlinear simulation can be explained by the impact of the subdominant TEM in the nonlinear saturated state. In this context, we underline that the investigation of off-diagonal impurity transport components can play a particularly interesting role in diagnosing the transport properties of a turbulent state. In fact, at comparable ion to electron heat fluxes, conditions can be achieved where the dominant mode produces a much smaller impurity convection than the subdominant mode (in absolute value), with the consequence that the convective component provided by the subdominant mode 
produces the dominant contribution in the nonlinear state. Thereby, the direction of the impurity convection becomes a clear macroscopic fingerprint of the non-negligible role of the subdominant linear mode (or, more in general, of the interplay of dominant and subdominant linear modes) in the nonlinear saturated turbulent state.

This theoretical result is also of practical interest. Centrally hollow impurity density profiles are highly desirable, particularly in proposed reactor scenarios where impurity seeding is applied in order to radiate part of the power in the peripheral region of the plasma before reaching the walls. As long as the radiated power is peripheral, this has limited impact on the plasma stored energy, as experimentally observed [57] and as can be expected by generic arguments based on temperature profile stiffness [49]. In contrast, central radiation cannot be tollerated in a reactor, as this heavily limits the possibilities of reaching ignition or large multiplication factors of the power. The nonlinear gyrokinetic results presented in this work show that, thanks to the contribution of subdominant modes, significant outward convection of heavy impurities is obtained in a range of electron to ion heat flux ratios which is significantly broader than that obtained by the linear predictions based on the dominant linear mode only. Reactor relevant conditions, in which the electron heat flux is equal to (or slightly exceeds) the ion heat flux, are consistent with a reduced inward convection (at low toroidal rotation) or an outward convection (at moderate $M_{D} \approx 0.15$ ), whereas are predicted to be characterized by a (stronger) inward convection in the predictions based on the dominant linear mode.

These theoretical results of heavy impurity transport obtained with nonlinear gyrokinetic simulations await experimental validation. Experiments in which the turbulent transport of heavy impurities is maximized with respect to the neoclassical transport, by combining high heating power levels, in the appropriate range of electron to ion heat flux ratio $1 \leq Q_{e} / Q_{i} \leq 2$, with the absence of significant torque applied to the plasma (and thereby with limited toroidal rotation), would be particularly appropriate to this purpose.

\section{Acknowledgments}

One of the Authors (C.A.) would like to thank R. Buchholz and W. Hornsby for their kind and valuable help in the utilization of the code GKW. This work has been carried out within the framework of the EUROfusion Consortium and has received funding from the Euratom research and training programme 2014-2018 under grant agreement No 633053 and from the RCUK Energy Programme [grant number EP/1501045]. The views and opinions expressed herein do not necessarily reflect those of the European Commission.

[1] Fussmann, G, Hofmann, J.V, Janeschitz G. et al 1989 J. Nucl. Mat. 162-164 14.

[2] Ida K., Fonck R.J, Sesnic S., Hulse R.A., LeBlanc B. and Paul S.F. 1989 Nucl. Fusion 29231.

[3] Fussmann G., Field A.R., Kallenbach A., Krieger K., Steuer K.-H. and the ASDEX Team 1991 Plasma Phys. Control. Fusion 331677. 
Gyrokinetic study of turbulent convection of heavy impurities in tokamak plasmas at comparable ion and

[4] Perry M.E., Brooks N.H., Content D.A., Hulse R.A., Ali Mahdavi M. and Moos H.W. 1991 Nucl. Fusion 311859.

[5] Pasini D., Giannella R., Lauro Taroni L., Mattioli M., Denne-Hinnov B., Hawkes N., Magyar G. and Weisen H. 1992 Plasma Phys. Control. Fusion 34677.

[6] Rice J.E., Goetz J.A., Granetz R.S., Greenwald M.J., Hubbard A.E., Hutchinson I.H., Marmar E.S., Mossessian D., Sunn Pedersen T., Snipes J.A., Terry J.L. and Wolfe S.M. 2000 Phys. Plasmas 71825.

[7] Rice J.E., Bonoli P.T., Marmar E.S., Wukitch S.J., Boivin R.L., Fiore C.L., Granetz R.S., Greenwald M.J., Hubbard A.E., Hughes J.W., Hutchinson I.H., Irby J.H., Lin Y., Mossessian D., Porkolab M., Schilling G., Snipes J.A. and Wolfe S.M. 2002 Nucl. Fusion 42510.

[8] Dux R., Neu R., Peeters A.G., Pereverzev G., Mück A., Ryter F., Stober J., Plasma Phys. Control. Fusion 45, 1815 (2003).

[9] Rice J.E. et al 2007 Fusion Sci. Technol. 51357.

[10] Pütterich T.et al 2013 Plasma Phys. Control. Fusion 55124036.

[11] Angioni C. et al 2014 Nucl. Fusion 54083028.

[12] Casson F. J. et al 2015 Plasma Phys. Control. Fusion 57014031.

[13] Angioni C. et al 2015 Phys. Plasmas 22055902.

[14] Neu R. et al 2003 J. Nucl. Mater. 313116.

[15] Fröjdh M., Liljestrom M., Nordman H, Nucl. Fusion 32, 419 (1992).

[16] Angioni C. and Peeters A.G. 2006 Phys. Rev. Lett. 96095003.

[17] Dubuit N., Garbet X., Parisot T., Guirlet R., and Bourdelle C. 2007 Phys. Plasmas 14042301.

[18] Angioni C. et al 2007 Phys. Plasmas 14055905.

[19] Nordman H. et al 2008 Phys. Plasmas 15042316.

[20] Camenen Y., Peeters A.G., Angioni C. et al 2009 Phys. Plasmas 16012503.

[21] Fülöp T., Braun S., and Pusztai 2010 Phys. Plasmas 17062501.

[22] Fülöp T. and Moradi S. 2011 Phys. Plasmas 18030703.

[23] Skyman A. 2012 Phys. Plasmas 19032313.

[24] Mollen A. et al 2012 Phys. Plasmas 19052307.

[25] Angioni C. et al 2012 Phys. Plasmas 19122311.

[26] Angioni C. et al 2012 Nucl. Fusion 52114003.

[27] Puiatti M.E. et al 2006 Phys. Plasmas 13042501.

[28] Angioni C. et al 2007 Plasma Phys. Control. Fusion 492027.

[29] Nordman H et al 2011 Plasma Phys. Control. Fusion 53105005.

[30] Angioni C. et al 2011 Nucl. Fusion 51023006.

[31] Valisa M. et al 2011 Nucl. Fusion 51033002.

[32] Howard N.T. et al 2012 Nucl. Fusion 52063002.

[33] Howard N.T. et al 2012 Phys. Plasmas 19056110.

[34] Casson F.J. et al 2013 Nucl. Fusion 53063026.

[35] Mollén A. et al 2014 Plasma Phys. Control. Fusion 56124005.

[36] Henderson S.S. et al 2015 Plasma Phys. Control. Fusion 57095001.

[37] Angioni C. 2015 Phys. Plasmas 22120501.

[38] Peeters A.G. et al 2009 Comput. Phys. C. 1802650.

[39] Casson F. J. et al 2010 Phys. Plasmas 17102305.

[40] Hirshman S.P. and Sigmar D.J. 1981 Nucl. Fusion 211079.

[41] Wong S.K. 1987 Phys. Fluids 30818.

[42] Romanelli M., Ottaviani M. 1998 Plasma Phys. Control. Fusion 401757.

[43] Fülöp T. and Helander P. 1999 Phys. Plasmas 63066.

[44] Angioni C, Helander P. 2014 Plasma Phys. Control. Fusion 56, 124001.

[45] Belli E., Candy J., Angioni C. 2014 Plasma Phys. Control. Fusion 56, 124002.

[46] ITER Physics expert groups on confinement and transport and confinement modelling et al 1999 Nucl. Fusion 392175.

[47] Valisa M. et al 2014 Proceedings of the $25^{\text {th }}$ IAEA Fusion Energy Conference 2014, Saint 
Gyrokinetic study of turbulent convection of heavy impurities in tokamak plasmas at comparable ion and

Petersburg, Russia.

[48] Mantica P. et al 2015 EPS Conference on Plasma Physics, Lisbon, Portugal.

[49] Wenninger R. et al 2014 Nucl. Fusion 54114003.

[50] E. Fable, 2016 Nucl. Fusion submitted (Special Issue on 15th International Workshop on H-mode Physics and Transport Barriers, Garching, Germany).

[51] Merz F. and Jenko F. 2010 Nucl. Fusion 50054005.

[52] Angioni C., Peeters A.G., Pereverzev G.V., Bottino A., Candy J., Dux R., Fable E., Hein T. and Waltz R.E. 2009 Nucl. Fusion 49055013.

[53] Buckholz R. 2016 University of Bayreuth, Ph. D.

[54] Staebler G.M. et al 2007 Phys. Plasmas 14055909.

[55] Halpern F.D., Eriksson A., Bateman G., Kritz A.H., Pankin A.Y., Wolfe C.M. and Weiland J. 2008 Phys. Plasmas 15012304

[56] Bourdelle C., Garbet X., Imbeaux F. et al 2007 Phys. Plasmas 14112501.

[57] Kallenbach A. et al 2012 Nucl. Fusion 52122003. 